\title{
Essential syphilitic alopecia: Non-scarring alopecia in 21-year-old man
}

\section{Hafssa Chehab, Bertrand Richert}

\author{
Department of Dermatology, Brugmann University Hospital, Brussels, Belgium
}

Corresponding author: Dr. Hafssa Chehab, E-mail: hafssachehab@gmail.com

\begin{abstract}
Alopecia syphilitica is a less common clinical manifestations of secondary syphilis. It is uncommon for hair loss to be the sole or predominant manifestation, as hair loss is the chief clinical and histologic differential diagnosis of. The main difference between alopecia areata and Alopecia syphilitica is the detection of Treponema pallidum in syphilis. We present the case of a 2l-year-old belgium man with different patches of non-cicatricial alopecia of his scalp. The patient denied previous history of genital or other skin lesions. Laboratory evaluation was positive for syphilis. The diagnosis of alopecia syphilitica was made and he was treated with single intramuscular injections of benzathine penicillin. The lesions improved with treatment in all the patients who attended follow-up. Dermatologists should maintain a high level of clinical suspicion for this uncommon manifestation of syphilis, particularly when it is the only symptom.
\end{abstract}

Key words: Alopecia; non-cicatricial; Treponema pallidum

\section{INTRODUCTION}

Loss of hair on the scalp or elsewhere on the body of persons with secondary syphilis is called syphilitic alopecia. It is one of the clinical manifestations of secondary syphilis.

The characteristic "moth-eaten" appearance on the scalp has been described by many authors.

In 1940, McCarthy described two types of syphilitic alopecia, symptomatic alopecia, associated with other lesions of secondary syphilis; essential syphilitic alopecia, which has no other cutaneous or mucosal manifestations of the disease [1].

Alopecia syphilitica is reported in the literature, but it is uncommon for hair loss to be the only or predominant manifestation [2].

\section{CASE REPORT}

A 21-year-old male presented with hair loss for about 2 months with ineffective treatement with minoxidil and local corticosteroids.
Physical examination revealed multiple plaques of nonscarring alopecia, predominantly affecting the parietooccipital region. The plaques varied in size, did not present signs of inflammation or desquamation, and were not completely devoid of hair they had the characteristic moth-eaten or patchy appearance (Fig. 1).

Complete blood count and platelets, basic serum chemistries, liver function tests, thyroid function tests, and anti-nuclear antibodies were within normal limits or absent; his Venereal disease research laboratory test (VDRL) and Treponema pallidum hemagglutination assay (TPHA) was extremely reactive. Testing to determine the presence or absence of human immunodeficiency virus (HIV) was recommended. However, the patient refused further evaluation.

The diagnosis of alopecia syphilitica was made and he was treated with single intramuscular injections of benzathine penicillin (2.4 million units). Two months later VDRL level and alopecia improved.

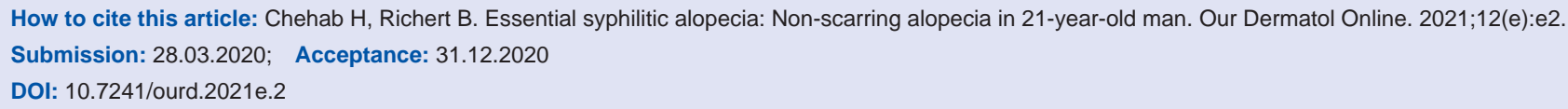




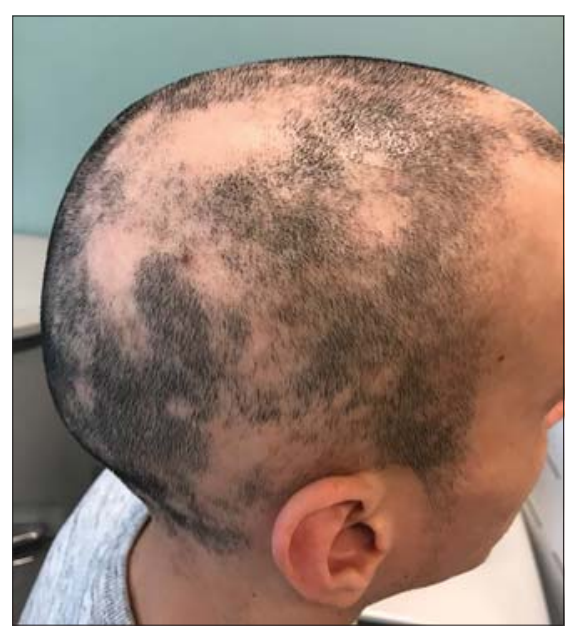

Figure 1: Scattered plaques of nonscarring alopecia.

\section{DISCUSSION}

SA is an unusual manifestation that only occurs in $4 \%$ of cases of syphilis [3].

Alopecia syphilitica is non-inflammatory and noncicatricial hair loss associated with secondary syphilis. It can present either by itself as "essential syphilitic alopecia," or with other symptoms of secondary syphilis, as "symptomatic syphilitic alopecia" [4].

Symptomatic SA is an extremely rare manifestation in which there are associated skin lesions on the scalp, usually in the form of papulosquamous lesions. The second type, essential SA, is characterized by hair loss with no other visible syphilitic lesions on the scalp.

Patients with alopecia syphilitica have also been reported in whom hair loss occurred on the eyebrows, eyelashes, chest, legs, axilla, and pubis [4].

\section{Essential SA has 3 distinct clinical patterns [4]:}

Moth-eaten or patchy which is characterized by the presence of small scattered plaques of nonscarring alopecia that are not completely hairless and show no signs of inflammation or desquamation. They occur mainly in the parieto-occipital region, possibly due to a greater load of treponemes because of the rich local blood supply to this area.

Diffuse: In this pattern, alopecia is diffuse, caused by widespread telogen effluvium-type hair loss, and there are no obvious plaques.

Mixed: Small, irregular plaques of alopecia develop on a background of diffuse alopecia.
The moth-eaten pattern is the pathognomonic of presentation on the scalp and is considered one of the most characteristic signs of secondary syphilis [5].

At dermoscopy, the typical signs described in the literature, such as vellus hairs, empty hair follicles, follicular hyperkeratosis, peripheral black spots, hypopigmented hairs, and dilated and tortuous vessels were visualized on an erythematous-brown background [5].

In addition, syphilis must be included in the differential diagnosis when patients have rapid diffuse hair loss of unknown etiology [6].

SA can be associated with systemic manifestations such as general malaise, low-grade fever, lymphadenopathies, asthenia, anorexia, and various mucosal and cutaneous manifestations [7].

SA should be suspected clinically and confirmed by laboratory studies such as serology (reagin and antitreponemal antibodies), which is always positive in secondary syphilis [7].

The histology of SA lesions usually shows preservation of the epidermis with areas of follicular hyperkeratosis. Psoriasiform epidermal hyperplasia with a lichenoid infiltrate has occasionally been reported [6].

The pathogenesis of alopecia syphilitica has not been elucidated due to failure to consistently demonstrate the presence of spirochetes in hair follicles.

In 2006, Nam-Cha et al. detected T. pallidum in an immunohistochemical study of a biopsy of a 24-year-old, HIV positive Hispanic man with alopecia syphilitica. The spirochete was detected in the peribulbar region and penetrated into the follicle matrix. This was the first time that organisms were detected in hair follicles, supporting the speculation that T. pallidum may be responsible for alopecia syphilitica [8].

The treatment of choice in immunocompetent individuals is an intramuscular dose of 2.4 million units of penicillin $\mathrm{G}$ benzathine [3]. In HIV-infected patients, weekly doses must be administered for 3 weeks.

SA may sometimes be the only manifestation of secondary syphilis, as in our case, and it is therefore important to maintain a high level of diagnostic suspicion in order to avoid a late or incorrect diagnosis. 


\section{Consent}

The examination of the patient was conducted according to the Declaration of Helsinki principles.

The authors certify that they have obtained all appropriate patient consent forms. In the form the patient(s) has/have given his/her/ their consent for his/her/their images and other clinical information to be reported in the journal. The patients understand that their names and initials will not be published and due efforts will be made to conceal their identity, but anonymity cannot be guaranteed.

\section{REFERENCES}

1. McCarthy L. Diagnosis and treatment of diseases of the hair. St Louis, MO: CV Mosby. 1940:537. n.d.

2. Schlupen E-M. Alopecia specifica in secondary syphilis. Molecular detection of treponema pallidum in lesional skin. Eur J Dermatol. 1996;6:19-22.
3. Chiu H-H, Wu C-S. Alopecia syphilitica. Indian J Sex Transm Dis AIDS. 2017;38:192-3.

4. Doche I, Hordinsky MK, Valente NYS, Romiti R, Tosti A. Syphilitic alopecia: case reports and trichoscopic findings. Skin Appendage Disord. 2017;3:222-4.

5. Bomfim ICC, Ianhez M, Miot HA. Dermatoscopic findings of syphilitic alopecia. An Bras Dermatol. 2020;95:518-20.

6. Tognetti L, Cinotti E, Perrot J-L, Campoli M, Rubegni P. Syphilitic alopecia: uncommon trichoscopic findings. Dermatol Pract Concept. 2017;7:55-9.

7. Hernández-Bel P, López J, Sánchez JL, Alegre V. Sífilis secundaria nodular. Actas Dermo-Sifiliográficas. 2009;100:520-2.

8. Nam-Cha SH, Guhl G, Fernández-Peña P, Fraga J. Alopecia syphilitica with detection of Treponema pallidum in the hair follicle. J Cutan Pathol. 2007;34 Suppl 1:37-40.

Copyright by Hafssa Chehab, et al. This is an open-access article distributed under the terms of the Creative Commons Attribution License, which permits unrestricted use, distribution, and reproduction in any medium, provided the original author and source are credited.

Source of Support: Nil, Conflict of Interest: None declared. 